\title{
Morfología urbana de Madrid, Cundinamarca
}

Urban morphology of Madrid, Cundinamarca

\author{
William Antonio Aponte Rodríguez ${ }^{1}$
}

\section{Resumen}

Este artículo presenta el estudio del proceso de urbanización de Madrid (Cundinamarca), desde la perspectiva que aporta la morfología urbana. Para ello se investigaron las variables emplazamiento, situación y plano, las cuales fueron operativizadas cualitativa y cuantitativamente, a fin de generar indicadores que permitieran establecer el estado actual y las tendencias de crecimiento de la ciudad. Los resultados obtenidos muestran como dichas variables han influenciado la forma física, la estructura interna y la expansión de la ciudad en un contexto en el que la metropolización de Bogotá, refuerza la influencia de estas variables.

Palabras clave: Cundinamarca, Madrid, morfología urbana.

\begin{abstract}
This paper presents the study of the process of the urbanization of Madrid (Cundinamarca), from the perspective provided by the urban morphology. With this purpose we investigated the following variables: location, situation and urban plan, which were made operational qualitatively and quantitatively to generate indicators to establish the current status and trends of growth of the city. The results show how these variables have influenced the urban form, the internal structure and the expansion of the city in a context in which the metropolization of Bogotá reinforces the influence of these variables.
\end{abstract}

Key words: Cundinamarca, Madrid, urban morphology. 


\section{Introducción}

La dinámica de urbanización en la región denominada Sabana de Bogotá es un tema que despierta gran interés dada su magnitud y los impactos que implica a nivel regional, departamental y nacional. Molina (2003) señala que las tendencias de este fenómeno apuntan a que en el año 2020 el conjunto urbano integrado por Bogotá, Soacha, Chía, Funza, Madrid y Mosquera conformará una inmensa conurbación de más de 10 millones de habitantes, concentrando casi una cuarta parte de la población nacional. A esto se suma la forma como ha evolucionado la distribución espacial de la población en la región.

Las cifras del Departamento Administrativo Nacional de Estadística DANE indican un proceso de descentralización demográfica respecto a Bogotá, que coincide con la concentración poblacional en los núcleos urbanos más próximos a la capital. Este comportamiento, que se corresponde con las dinámicas demográficas de las grandes metrópolis mundiales, evidencia la importancia que adquieren las ciudades intermedias ${ }^{2}$ como elemento

2 El concepto de ciudad intermedia, como el concepto mismo de ciudad, es difuso y complejo. Sin embargo existe consenso científico para definir la ciudad en torno a características como la población que alberga y la función que cumple en el sistema urbano (Beaujeu-Garnier, 1970). La UNESCO aplica este criterio, generalizando el concepto de ciudad intermedia para aquellos conglomerados urbanos que albergan poblaciones entre 20.000 y 2 millones de habitantes. Este criterio poblacional es complementado con características relativas a su rol o función: nodos urbanos de jerarquía intermedia integrantes de sistemas urbanos que se conectan a través de infraestructuras locales, regionales y nacionales (con acceso a infraestructuras internacionales como el caso de las ciudades periféricas a las urbes metropolitanas), centros proveedores de bienes y servicios especiali- del sistema urbano con gran potencial para equilibrar las relaciones territoriales a nivel regional (UNESCO, 1999), justificando la necesidad de emprender investigaciones que ayuden a ampliar el conocimiento que se tiene sobre ellas.

Madrid (Cundinamarca), ciudad intermedia localizada a $12 \mathrm{~km}$ de Bogotá (Colombia), en el segundo anillo metropolitano, es un ejemplo de las dinámicas urbanas mencionadas en el anterior párrafo. Los datos de los últimos cinco censos de población y vivienda, realizados por el DANE (1951, 1973, 1985, 1993 y 2005), indican que desde la segunda mitad del siglo XX, esta ciudad ha mantenido tasas de urbanización que superan a las de Colombia, Cundinamarca y Bogotá. $\mathrm{Su}$ importancia, como punto de acogida de aquellos pobladores que buscan localizarse en la región metropolitana, escapando de las externalidades negativas de la concentración en la gran ciudad (aglomeración, inseguridad, contaminación ambiental, altos costos de vivienda y problemas de movilidad), se ve reforzada por las tendencias de diversificación funcional, resultado del surgimiento de nuevos usos del suelo de vital importancia para la región como grandes superficies para bodegaje, logística y vivienda para población de estratos altos, actividades que se suman a las ya existentes (industria, vivienda obrera y floricultura).

zados para la región, que satisfacen demandas de la población, alojan actividades de administración a nivel local o regional, escenario de la interacción social, económica y cultural de las zonas en que ejercen influencia directa y centros de explotación de amplias áreas rurales (UNESCO, 1999). 
Este contexto en el que se desarrolla la ciudad de Madrid da lugar a formular las siguientes preguntas:

¿Cómo ha sido el proceso de urbanización en esta ciudad intermedia?

¿Cuáles han sido los principales factores que han incidido en el proceso de urbanización de Madrid?

¿Cómo es la morfología urbana y la estructura interna de esta ciudad?

¿Cuáles son las tendencias del proceso de urbanización de Madrid?

Al buscar respuesta a estas preguntas, la revisión bibliográfica permite identificar la existencia de gran cantidad de estudios, realizados desde diversas perspectivas (económica, sociológica, urbanística, geográfica y política entre otras), sobre el proceso de urbanización en la Sabana de Bogotá. Sin embargo, la gran mayoría de estos estudios centran su atención en la ciudad capital, tratando el tema de las ciudades intermedias de manera muy superficial. A esto se suma que existen muy pocos estudios que aborden el proceso de urbanización de ciudades intermedias de la Sabana de Bogotá desde una perspectiva geográfica y mucho menos para la ciudad de Madrid.

Esta ausencia de estudios sobre el tema motivó la ejecución de un proyecto de investigación cuyo objetivo principal apunta a conocer con mayor detalle el proceso de urbanización de Madrid durante las últimas cuatro décadas, desde la mirada que aporta la subdisciplina de la geografía urbana. Este artículo sintetiza algunos apartes de dicha investigación.
El documento aborda el proceso de urbanización de Madrid a partir de los conceptos desarrollados por la morfología urbana. Este campo de investigación de la geografía permite indagar sobre la forma urbana considerándola como una construcción social que, como lo exponen Capel (2002), Lévy (2005) y Allain (2004), refleja la organización económica, la estructura política y social, los objetivos de las clases dominantes y los acuerdos y conflictos de los distintos actores que han construido la ciudad. El estudio de la morfología urbana persigue intereses tanto descriptivos, como explicativos e interpretativos, ya que permite entender la producción del tejido urbano a partir de los elementos que lo constituyen y los mecanismos de transformación que intervienen en su desarrollo. También facilita el diseño de la ciudad por medio de un conocimiento profundo de su evolución, su composición interna y su estado actual, con una mirada multiescalar.

La investigación está orientada a partir de los principios teóricos que se acaban de exponer. Para ello se estudian los conceptos de emplazamiento, situación y plano, provenientes de la morfología urbana, los cuales son implementados como variables que permiten comprender el proceso de expansión de la ciudad de Madrid, su actual composición interna y la forma de su tejido urbano. Esto se logra con la medición de una serie de indicadores como la estratificación socioeconómica, la fragmentación de las parcelas urbanas y la localización de los usos del suelo urbano que, complementados con una detallada contextualización geográfica de 
la ciudad, facilitan la identificación de las formas urbanas dominantes en la ciudad y la composición de su estructura interna.

El estudio arroja como resultado que la actual morfología urbana de la ciudad de Madrid es producto de una serie de factores de tipo local y regional, como la situación geográfica en el contexto histórico, su atractivo como núcleo urbano generador de empleo, su proximidad a Bogotá y la localización puntual de equipamientos y actividades institucionales e industriales de gran magnitud, que en conjunto han determinado las formas de ocupación del espacio urbano.

\section{Localización geográfica de la ciudad de Madrid}

Madrid se localiza en el departamento de Cundinamarca, exactamente en la subregión occidental de la Sabana de Bogotá, en el primer anillo metropolitano de Bogotá (Molina, 2003). El municipio es atravesado por dos importantes vías de orden regional y nacional, que comunican a la capital con el occidente, la autopista Medellín y la Troncal de Occidente, y por la vía férrea que va de Bogotá a Puerto Salgar la cual va paralela a la Troncal de Occidente en su paso por el municipio. La ciudad de Madrid se ubica a $29 \mathrm{~km}$ de Bogotá, sobre la Troncal de Occidente y la mencionada línea férrea. En el municipio de Madrid se distinguen dos zonas geográficas claramente diferenciadas: una zona plana que corresponde al $84 \%$ de la extensión del municipio, y sobre la cual se localiza la ciudad. Esta zona se caracteriza por un relieve plano, poco inundable por las óptimas condiciones de drenaje, con buena disponibilidad de aguas superficiales ${ }^{3}$ y subterráneas ${ }^{4}$ (gracias a la presencia de acuíferos debidos a las condiciones hidrogeológicas de esta zona), suelos fértiles y profundos. Todas estas condiciones configuran esta zona como ideal para el desarrollo de actividades agropecuarias. La segunda zona, la cual ocupa el $16 \%$ restante de la extensión territorial del municipio, está conformada por una elevación montañosa que alcanza los 2.875 metros de altura, por encima de los $2.600 \mathrm{msnm}$ que en promedio alcanza la Sabana en esta zona, y que se ubica al sur de la cabecera urbana. La Figura1 permite apreciar la localización de la ciudad de Madrid a nivel nacional, departamental y municipal.

\section{Metodología}

Este estudio está enmarcado en un diseño metodológico de tipo descriptivo, que partió de la revisión bibliográfica para identificar técnicas y enfoques que permitieron concretar la investigación en tres variables: emplazamiento, situación y plano. Estas variables fueron estudiadas, medidas e interpretadas por medio de análisis operativizados de forma cuantitativa o cualitativa según el tipo de variable. Para ello se emplearon tres técnicas de análisis: análisis documental, análisis estadístico y análisis cartográfico.

3 El municipio de Madrid es atravesado por los ríos Subachoque y Bojacá.

4 Las condiciones hidrogeológicas de Madrid propician la existencia de acuíferos y aguas profundas. 
Figura 1. Localización de la ciudad de Madrid en la región Sabana de Bogotá
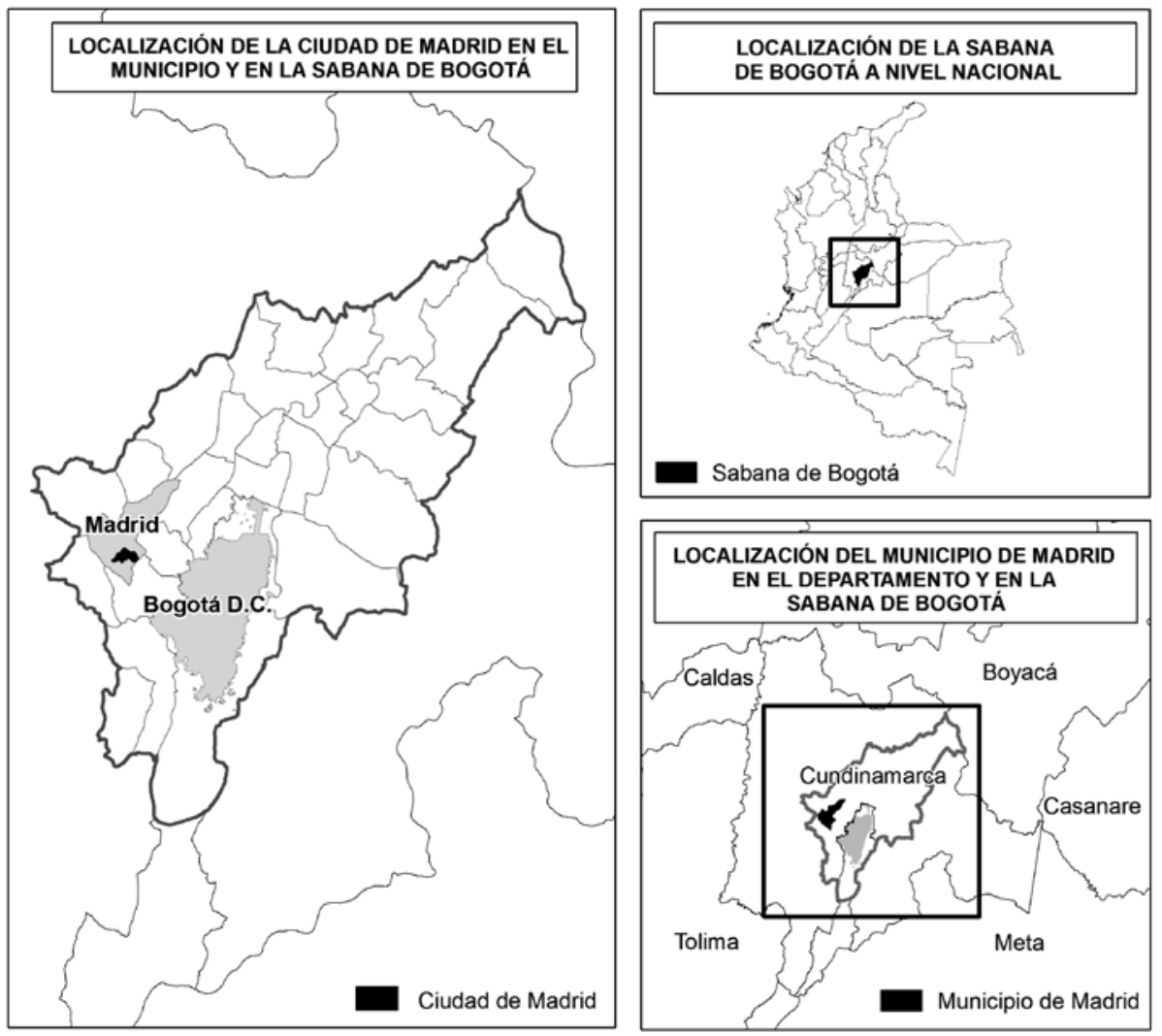

Fuente: elaboración propia con base en la cartografía IGAC escala 1:100.000.

En el análisis documental se identificó la convergencia de conceptos desarrollados por autores como Allain (2004), Capel (2002), Levy (2005) y Zárate (1992), quienes sostienen que las formas urbanas son un producto social cuyo conocimiento puede lograrse por medio de la investigación del tejido urbano, de los elementos que lo integran y de su relación con las condiciones socioeconómicas del espacio geográfico en que se localiza. A partir de esta premisa se seleccionaron las variables investigadas y se estableció la forma como fueron interpretadas o medidas dichas variables. Adicionalmente, el análisis documental permitió desarrollar el estudio de las variables emplazamiento y situación, las cuales fueron estudiadas cualitativamente.

El análisis estadístico fue empleado para medir los indicadores tamaño parcelario y superficie ocupada por usos del suelo, correspondientes a la variable plano, cuya operativización es de tipo cuantitativo. Para ello se utilizaron técnicas de 
estadística descriptiva que permitieron la organización, tabulación, presentación e interpretación del conjunto de datos mediante tablas e histogramas en los que se aprecia la magnitud de los indicadores estudiados. Estos indicadores fueron georreferenciados con el fin de establecer su comportamiento espacial. Dicha tarea se ejecutó empleando técnicas de procesamiento y análisis para información espacial por medio de herramientas SIG. Por último, se fijó el estado actual de la ciudad a partir del análisis conjunto de las variables investigadas y de los resultados obtenidos.

La escala espacial fue local (1:20.000), para lo cual se emplearon unidades espaciales de análisis como la cabecera urbana, la manzana y el lado de manzana. Sin embargo, los análisis de las variables emplazamiento y situación requirieron análisis a escala regional $(1: 100.000 \mathrm{y}$ 1:500.000) para lograr su correcta descripción, ya que los elementos que las definen y determinan superan la escala local. En cuanto a la escala temporal, teniendo en cuenta que se caracterizaron las condiciones actuales de la ciudad, el estudio se considera de tipo sincrónico. Sin embargo fueron incluidos ciertos factores históricos que determinan las formas urbanas en la actualidad, por lo que, para la variable emplazamiento, se emplearon análisis de tipo diacrónico.

Considerando los enfoques cognitivo y normativo definidos por Gauthier y Jason $(2006)^{5}$ el estudio que aquí se

5 Según estos autores, el enfoque cognitivo hace referencia al esclarecimiento de fundamentos filosóficos y epistemológicos que sustenten el estudio de la morfología urbana, presenta se puede clasificar en el enfoque normativo ya que se trata de una investigación aplicada que tiene por objeto investigar las condiciones de una ciudad en particular y no el desarrollo de teorías o métodos.

La variable emplazamiento fue estudiada partiendo de la definición dada por Puyol et al. (1988). Según estos autores, el emplazamiento es el espacio concreto y material sobre el que se asienta la ciudad. Zárate (1991) amplía esta definición indicando que el emplazamiento está conformado por elementos como la geología, el relieve, el clima, la hidrología, los suelos y la vegetación, los cuales determinan el establecimiento de la ciudad, la forma y dimensiones de la expansión física, así como las características del paisaje urbano. Lo expuesto por Capel (2002), quien resalta el papel del emplazamiento como el espacio geográfico previamente seleccionado que satisface ciertas necesidades a partir de las cuales se funda el núcleo urbano y las cuales determinan las funciones urbanas originales, complementa la definición de esta variable.

Para Zárate (1992), la situación es “... el entorno geográfico más amplio en el que la ciudad se enmarca y con relación al cual se organiza y...hace referencia... sobre todo, a vías de circulación, de intercambio y elementos de producción". La decisión de localización se basa en

así como al desarrollo de marcos explicativos y modelos teóricos, mientras que el enfoque normativo se orienta a la acción, es decir, al conocimiento y descripción de las formas urbanas con el fin de orientar los procesos de gestión y planificación. 
“...el deseo de dominar rutas de desplazamiento de hombres y mercancías, y por el anhelo de beneficiarse de la relación entre áreas de economía distinta y del contacto de sistemas de circulación diferentes". Bajo este criterio, el autor identifica cuatro tipos de situación: situación de encrucijada, situación en un eje de comunicaciones, situación en zona de contacto de áreas geográficas diferentes y situación de fachada marítima.

Para el estudio del plano se empleó el enfoque morfológico identificado por Carter (1974), y se analizan tres elementos que, según Capel (2002) y Lévy (2005), componen y describen el plano: el sistema viario, las manzanas y las parcelas individuales. Estos elementos fueron complementados con el análisis de la localización de usos del suelo.

\section{Resultados y discusión}

\subsection{Análisis del actual emplazamiento de la ciudad}

El emplazamiento de la ciudad de Madrid está determinado por la existencia de tres elementos naturales que han resultado fundamentales a lo largo de la evolución de esta ciudad.

El primero es la región geográfica conocida como Sabana de Bogotá, planicie de origen fluviolacustre (Van Der Hammen, 1998) ubicada en la cuenca alta del río Bogotá (Montañez, 1992), cuya extensión es de 425.160 ha (Pérez, 2000) y que se encuentra limitada por la cota $2.600 \mathrm{msnm}$. Como se ilustra en la Figura 1, Madrid se localiza en la zona occidental de esta región. Dicha ubicación ha permitido que en este municipio se presente una serie de características físicas, descritas más adelante, las cuales en conjunto, han configurado un escenario natural favorable para el desarrollo de actividades agropecuarias gracias a las ventajas comparativas que ofrece: topografía plana con pendientes que no superan el $3 \%$ y alturas que oscilan en promedio entre los 2.550 y $2.600 \mathrm{msnm}$ (IGAC, 2005); suelos de alto potencial agrícola $^{6}$ y una alta oferta hídrica gracias a la presencia de fuentes superficiales y a las condiciones hidrogeológicas que favorecen la existencia de abundantes aguas subterráneas aprovechables por medio de pozos y aljibes (alcaldía de Madrid, 2000).

El segundo elemento que determina el emplazamiento de Madrid es el río Subachoque $^{7}$ sobre cuya cuenca baja se localiza la ciudad (IGAC, 1974). Considerando los análisis sobre las lógicas de decisión de asentamiento que dan origen

6 Según la alcaldía de Madrid (1969), el 68,9\% de los suelos del municipio pertenecen a la Clase Agrológica I, apta para producir de buenas a excelentes cosechas y el 20,9\% pertenecen a las Clases II, III y IV, susceptibles de producir cosechas productivas con buenas prácticas de manejo. De esto se concluye que el $89,8 \%$ del área municipal ofrece un altísimo potencial para el desarrollo de actividades agrícolas y pecuarias.

7 El río Subachoque, cuyas aguas fluyen de norte a sur, nace en el Alto el Boquete, municipio de Subachoque, a una altura $3.700 \mathrm{~m}$ y desemboca en el río Balsillas en el mismo punto donde se encuentra con el río Bojacá, drenando una superficie de $450 \mathrm{~km} 2$, que comprende parte de los municipios de Subachoque, el Rosal, Madrid, Funza y Facatátiva. 
Figura 2. Situación y emplazamiento de la ciudad de Madrid a escala regional.

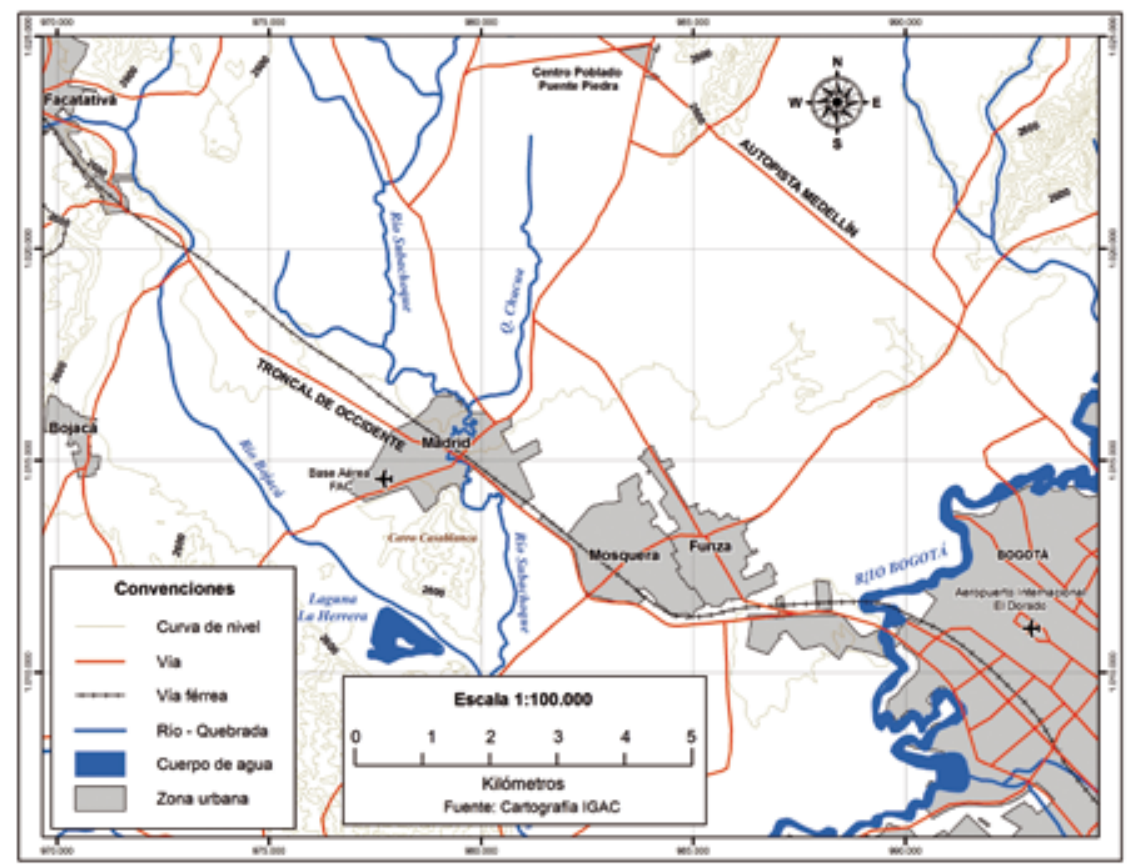

Fuente: elaboración propia con base en la cartografía IGAC escala 1:100.000.

a la fundación de núcleos urbanos descritas por Beaujeau (1970) y Capel (1975), y la localización del centro histórico, se puede inferir que el río Subachoque jugó un papel determinante en la elección del lugar en que se fundó el poblado que dio origen a la ciudad de Madrid ${ }^{8}$. Esta corriente hídrica proveyó el suministro de agua necesario para la subsistencia de los primeros grupos humanos que habitaron dicho poblado en la época de la Conquista.

8 Según relata Velandia (como se cita en Suárez, 1990), "Jiménez de Quesada (...) mandó a un capitán Vargas diciéndole estas palabras: "Id y fundad una población al pie de esa serranía", lo cual hizo, dándole el nombre de Serrezuela, por quedar junto a la única serranía de esta parte occidental de la Sabana. En 1542, las tierras de Serrezuela, fueron adjudicadas en encomienda al rodelero, o soldado de a pie".
El tercer elemento es el cerro Casablanca, elevación montañosa que se destaca en el paisaje sabanero, ya que abarca una extensión de 407 ha aproximadamente y alcanza una altura cercana a los 2.875 msnm, con pendientes superiores al 25 $\%$. Su localización ha sido un factor que ha frenado el crecimiento físico al sur de la ciudad y ha determinado el actual trazado del perímetro urbano como límite administrativo. Los actuales barrios vecinos a este cerro se han desarrollado preferentemente sobre zonas planas, lo que facilita el trazado y el proceso de asentamiento, por lo que el tejido urbano se ha adaptado a la forma perimetral de esta elevación montañosa. 


\subsection{Análisis de la situación de la ciudad}

La situación del poblado original, clasificada según Zárate (1992) como situación sobre un eje de comunicación, está dada por su ubicación con respecto al antiguo camino de Santafé de Bogotá a Honda y que hoy corresponde a la Troncal de Occidente. Según Delgado (2004), este camino jugó un papel económico determinante para consolidar a Santafé como capital del virreinato de la Nueva Granada, ya que le brindó una gran ventaja competitiva como punto de salida de la producción harinera del altiplano cundiboyacense por el río Magdalena, por tratarse de la ruta más corta y cuyo recorrido generaba los menores costos de transporte en comparación con la ruta Tunja, Vélez y el río Opón o el río Carare, la cual presentaba condiciones de infraestructura más ineficientes y una mayor longitud. Por el camino de Santafé a Honda salían y entraban mercancías y personas hacia la capital y fue por esta ruta por la que Humboldt hizo su ingreso a la capital en 1801. El poblado de Serrezuela, hoy Madrid, se benefició de esta localización ya que adquirió una función de relativa importancia no solo como lugar de paso sino como punto de aprovisionamiento, aclimatación y descanso para los funcionarios de la Real Audiencia, el arzobispo metropolitano y los virreyes, en sus visitas a Santafé de Bogotá (Suárez, 1990). Teniendo en cuenta que en ese entonces el transporte de carga se realizaba a "lomo de mula", por lo que factores como la distancia, la topografía y la calidad de los caminos jugaban un papel vital, y que la región de la Sabana era considerada la despensa de granos de la Nueva Granada, se concluye que este camino desempeñó una función determinante en el sistema vial de aquel entonces. Delgado (2004), en su investigación, afirma que desde el siglo XVII hasta el siglo XIX, esta ruta se mantuvo como la más "moderna" y la de menores costos de transporte para comunicar el altiplano cundiboyacense con el río Magdalena. El trazado ferroviario inaugurado en 1889, cuyo recorrido es paralelo a la ruta mencionada entre Bogotá y Facatativá, es otro elemento que reforzó la situación del núcleo urbano de Madrid, ya que en su momento fue punto nodal del Ferrocarril de la Sabana al establecerse allí una de sus estaciones.

En la actualidad, la situación de Madrid se encuentra determinada por su localización con respecto a la capital colombiana y al sistema urbano regional. En el contexto nacional y departamental, la ciudad se encuentra localizada sobre uno de los dos ejes viales, que convergen y se cruzan en Bogotá, comunicando a la capital y a la región de Cundinamarca con el resto del país: el primero es el eje norte-sur que conecta a Tunja con Girardot e Ibagué $y$ el segundo es el eje oriente-occidente que conecta a Villavicencio con Honda y con la zona cafetera (ver Figura 3). Estos ejes, a su vez, concentran la mayor cantidad de infraestructura de vías, equipamientos comunitarios y redes de servicios públicos sobre el territorio departamental (Mesa de Planificación Regional, 2005). 
En el contexto regional, Madrid se ubica en la zona con mayor presencia de infraestructura vial del país (Mesa de Planificación Regional, 2005). En esta zona, las condiciones topográficas y la cercanía a Bogotá han propiciado la configuración de una densa y compleja red vial que le permite a Madrid conectarse de forma fácil y rápida con todos los nodos que conforman el sistema urbano de la Sabana de Bogotá. Su localización es privilegiada si se tiene en cuenta que se ubica a una distancia de $12 \mathrm{~km}$ de Bogotá, ciudad capital que cuenta con la terminal aérea más importante del país, el aeropuerto Eldorado. Igualmente, Madrid se asienta sobre la Troncal de Occidente, vía de carácter nacional que comunica a la capital con el occidente de la región. Así mismo se encuentra conectada con la autopista Medellín, en un recorrido no mayor a $15 \mathrm{~km}$, a través de dos vías de carácter intermunicipal que conducen al centro poblado de Puente Piedra y de allí a Subachoque (ver Figura 2).

Figura 3. Situación de la ciudad de Madrid con respecto al sistema vial regional.

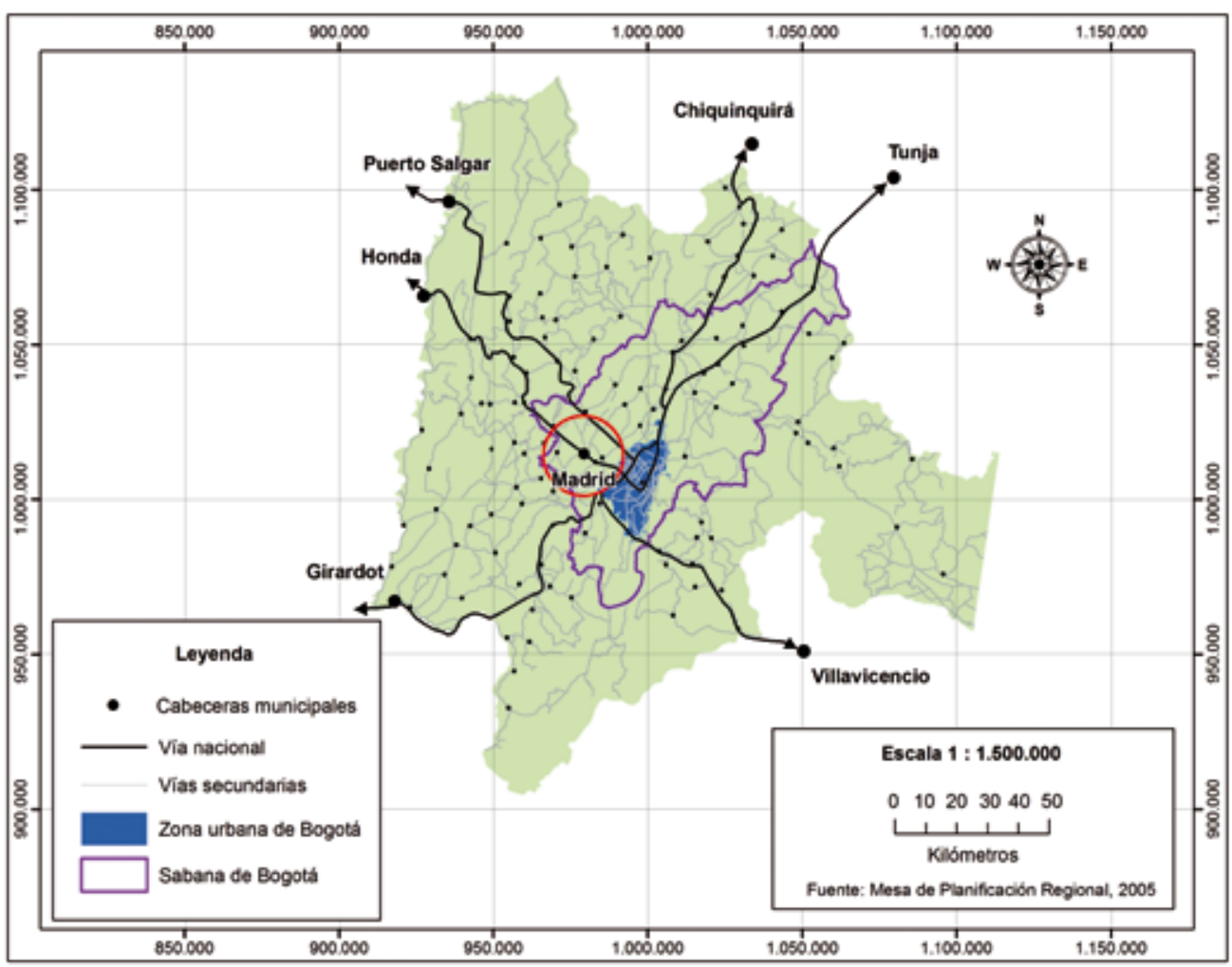

Fuente: Mesa de Planificación Regional (2005). 
Por otra parte, Madrid se ubica al interior del polígono metropolitano, sobre el eje occidental, en la zona de municipios claramente "metropolizados". Por su localización sobre la conurbación de occidente y su cercanía a la capital colombiana (ver Figura 2), Madrid, junto con Soacha, Chía, Funza y Mosquera, hace parte del conjunto de municipios que integran la megalópolis policéntrica de Bogotá (Mesa de Planificación Regional, 2005). Esto indica el alto grado de integración funcional existente entre Madrid y la capital, la cual se manifiesta principalmente en la alta dinámica demográfica de esta ciudad intermedia.

\subsection{Análisis geográfico del plano de la ciudad}

El plano actual de la ciudad es el resultado de un proceso de expansión que se ha dado a partir de la ubicación de su centro histórico. El principal elemento que ha configurado este proceso de expansión es la calle 7 , vía que corresponde a la Troncal de Occidente y que adquiere esta denominación a su paso por la ciudad. Esta vía, sobre la cual se localiza el centro histórico, constituye la columna vertebral del sistema vial de Madrid tanto a nivel urbano como rural. A partir de ella se desarrollaron los trazados que integran dicho sistema. Una mirada

Figura 4. Situación de la ciudad de Madrid con respecto a la infraestructura regional compuesta por el conjunto de vías, equipamientos comunitarios y redes de servicios públicos

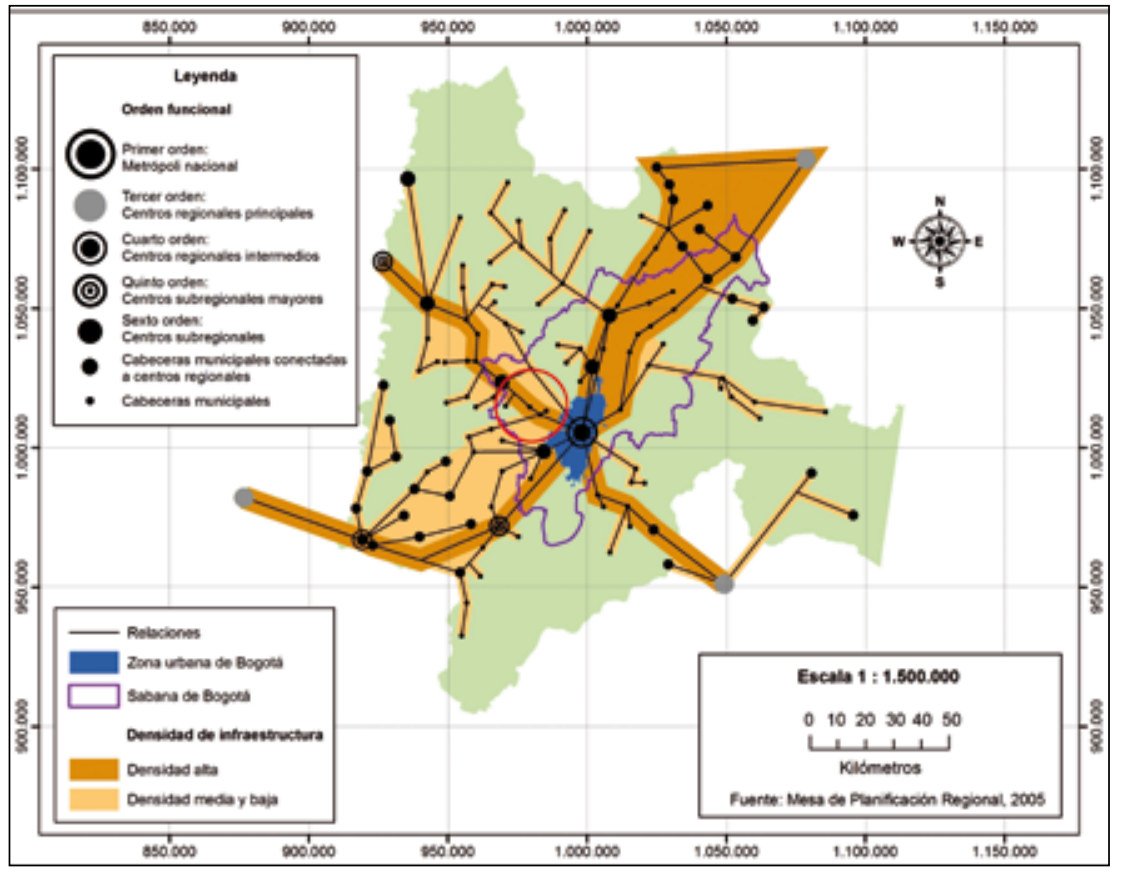

Fuente: Mesa de Planificación Regional (2005). 
global al conjunto de la ciudad permite apreciar que, como en muchas ciudades de Latinoamérica, la forma dominante para la conformación de las manzanas en la cabecera de Madrid es la de damero o cuadrícula. También se observa que este conjunto está compuesto por un mosaico de zonas que no presenta uniformidad. Esto se debe a la presencia de elementos naturales y culturales, a los cuales la expansión de la mancha urbana ha tenido que adaptarse, así como al desarrollo discontinuo, el cual corresponde al patrón de crecimiento urbano tipo "salto de rana" (leap-frog $)^{9}$, descrito por Benguigui et al. (2001), que se presentó desde la década del sesenta. Dicho proceso generó la existencia de espacios intersticiales o vacíos urbanos internos, que en la medida en que se han venido ocupando, han consolidado un tejido urbano heterogéneo, fragmentado y complejo, evidencia de los modelos que Torres (2005) identifica como productores de la ciudad y que Capel (2002) clasifica en la categoría de desarrollos reglados y desarrollos no reglados o espontáneos.

Uno de los elementos que por su forma, su destinación y su extensión ha contribuido a determinar la morfología urbana de Madrid en el cuadrante suroccidental de la ciudad, es la base aérea de la Fuerza Aérea Colombiana FAC. Esta base, de uso institucional, se localiza en el costado sur de la Troncal de Occidente y tiene una extensión aproximada de 90 ha, que corresponden al $13 \%$ del área de la ciu-

9 Este patrón de crecimiento corresponde a un desarrollo urbano discontinuo caracterizado por la localización de enclaves urbanos en la zona rural. dad $^{10}$. Por su carácter de zona militar ${ }^{11}$, el acceso y circulación por esta zona es restringido por lo que su localización constituye una barrera para la expansión urbana. Por lo tanto, la orientación de los trazados viales, y los desarrollos urbanísticos derivados de estos trazados, que se han localizado en la zona periférica a este sector, fueron realizados bordeando el perímetro de la base (carreras 9 y 20 al sur de la calle 7). Esto ha causado que el sector integrado por los barrios Sosiego, San Bernardo, San Carlos, Libertad y San José, ubicado al occidente de la ciudad, se encuentre completamente desarticulado y desconectado del conjunto urbano. (Ver Figura 5).

El otro elemento que constituye una barrera para la expansión de la ciudad es el cerro Casablanca. Como ya se había comentado en el numeral 3.1, esta elevación montañosa es la que determina el límite y la forma del perímetro urbano al sur de la ciudad, en la zona correspondiente al barrio las Hermandades, impidiendo el establecimiento de nuevos desarrollos urbanísticos en dicha zona.

Sumado a estos elementos modeladores del plano en la ciudad de Madrid, está el río Subachoque. Su recorrido, en dirección norte-sur, divide la ciudad en dos y se realiza describiendo una serie de sinuosidades en forma de meandros que en algunos tramos son bastante pronuncia-

10 La delimitación vigente del actual perímetro urbano, establecida según el Acuerdo 24 del 2000, por medio del cual se adopta el Plan Básico de Ordenamiento Territorial de Madrid, ocupa una superficie de 650 ha aproximadamente.

11 En las instalaciones de este complejo institucional, además de localizarse una pista aérea y varios talleres aeronáuticos, funciona la Escuela de Suboficiales CT Andrés M. Díaz. 
Figura 5. Desarrollos urbanísticos vecinos a la base aérea de la FAC

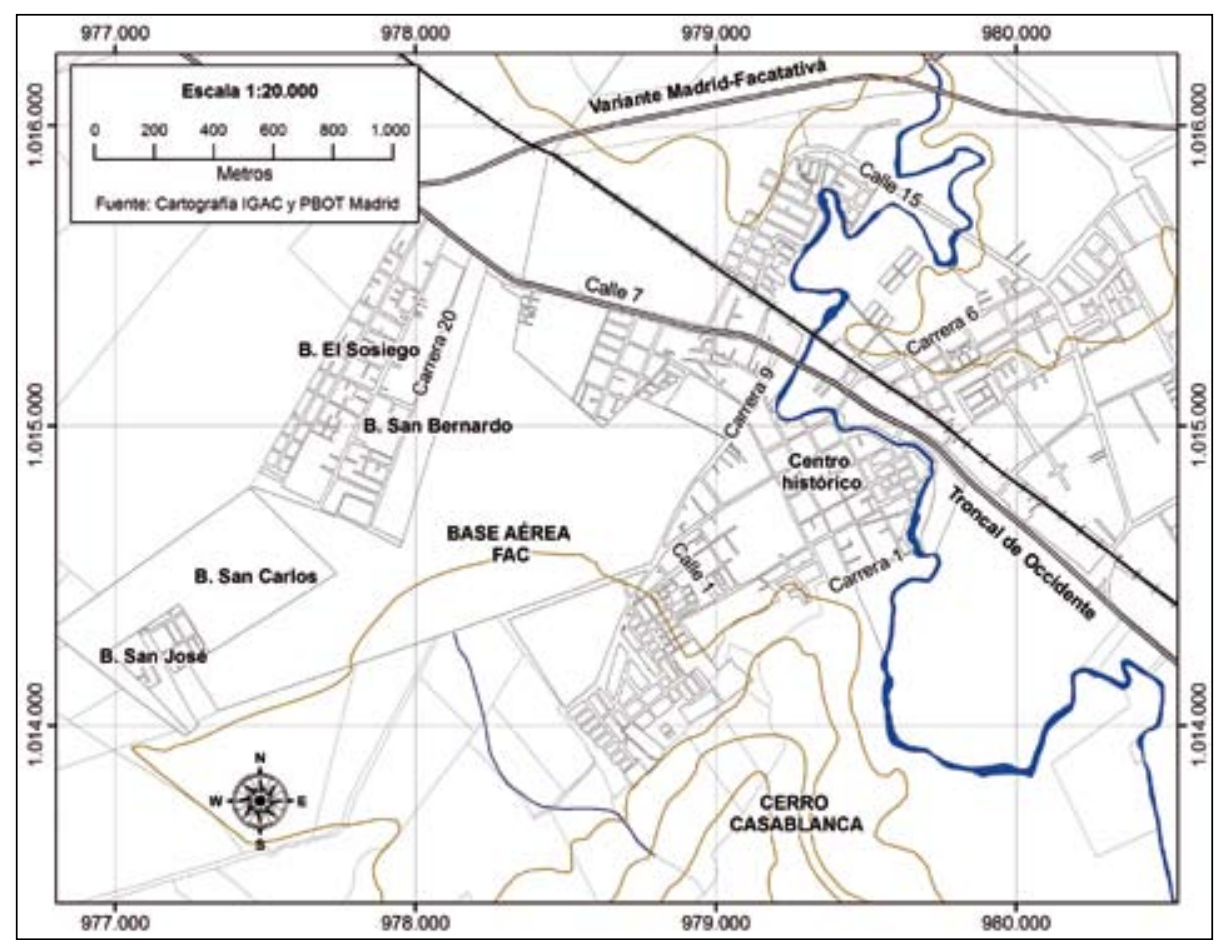

Fuente: elaboración propia a partir de la cartografía del PBOT de Madrid 2004.

dos. Como resultado, la continuidad del sistema vial y del plano resultante se ven interrumpidos abruptamente originando desarrollos caracterizados por geometrías irregulares que se han adaptado a la forma del río, con una lógica de máximo aprovechamiento del espacio edificable, de tal forma que la parte posterior de las edificaciones está dispuesta contra la ronda de este cuerpo hídrico.

El análisis parcelario, que Capel (2002) propone como enfoque descriptivo y explicativo de la evolución y composición interna de la ciudad, resulta complemen- tario a los elementos ya estudiados, para comprender el estado actual del plano de la ciudad de Madrid. La falta de registros catastrales históricos impide rastrear la forma como se ha dado la fragmentación de las parcelas originales. Sin embargo, la cartografía predial catastra ${ }^{12}$ permite cuantificar con precisión el estado actual de dicha fragmentación en la ciudad de Madrid. Para ello se establecieron rangos de área de parcela tomando

12 Para la realización del análisis parcelario en la ciudad de Madrid se tomó como insumo la cartografía predial catastral urbana suministrada por la alcaldía municipal y generada por la Subdirección de Catastro del IGAC para la vigencia 2009. 
como unidad de referencia el valor de $72 \mathrm{~m}^{2}$. La selección de este valor de referencia fue hecha considerando que los modelos de producción urbana para la vivienda destinada a la población de bajos ingresos, empleados en Madrid, son los mismos que se han utilizado en la ciudad de Bogotá (González, 2007) y que, según Tarchópolus y Ceballos (2005), la parcela de $72 \mathrm{~m}^{2}$ corresponde al patrón predominante en las tipologías edificatorias que caracterizan los desarrollos urbanísticos de vivienda de bajo costo en Bogotá ${ }^{13}$.

Los resultados de esta clasificación se pueden apreciar en la Tabla 1 y en la Figura 6. Los datos muestran que el 34,01 $\%$ de las parcelas corresponden a áreas inferiores a la unidad de referencia (72 $\mathrm{m}^{2}$ ) y ocupan el 3,7\% de la superficie total de la ciudad. Considerando que la mayoría de estas parcelas se ubica en zonas residenciales, se puede concluir que una tercera parte de las viviendas en la ciudad de Madrid se desarrolla en parcelas que están por debajo de los promedios para vivienda de bajos ingresos en Bogotá. Las parcelas de extensión superior a cuatro unidades de referencia $\left(288 \mathrm{~m}^{2}\right)$ que corresponden a una décima parte del total de parcelas, ocupan el 83,49\% de la superficie de la ciudad. Dentro de este rango existe un grupo de 67 parcelas (menos del $1 \%$ del total) que ocupan 321 ha $(63,64 \%$ del área total de la ciudad) de superficie. La localización de estas parcelas muestra que en su mayoría corresponden a las zonas ocupadas por la Base Aérea de la FAC y los desarrollos industriales existentes en la zona urbana. El conjunto de parcelas con áreas de hasta cuatro unidades de referencia (menores a $288 \mathrm{~m}^{2}$ ), y las cuales en su mayoría corresponden al uso residencial, corresponden al $90 \%$ del total de parcelas, ocupan el 16,51\% de la superficie y se distribuyen de forma fragmentada al interior de la cabecera urbana.

Tabla 1. Cantidad de parcelas y superficie ocupada por rangos de área en la ciudad de Madrid

\begin{tabular}{|c|c|c|c|c|c|}
\hline \multicolumn{2}{|c|}{ Rango de área } & \multicolumn{2}{c|}{ Predios } & \multicolumn{2}{c|}{ Superficie ocupada (m2) } \\
\hline $\begin{array}{c}\text { Unidades de } \\
\text { referencia }\end{array}$ & $\begin{array}{c}\text { Metros } \\
\text { cuadrados }\end{array}$ & Cantidad & $\%$ & Cantidad & $\%$ \\
\hline Menor a 1 & Hasta 72 m2 & 3.093 & 34 & 186.812 & 4 \\
\hline De 1 a 2 & De 73 a 144 m2 & 3.455 & 38 & 332.265 & 7 \\
\hline De 2 a 3 & De 145 a 216 m2 & 1.119 & 12 & 195.598 & 4 \\
\hline De 3 a 4 & De 217 a 288 m2 & 481 & 5 & 117.921 & 2 \\
\hline Mayor a 4 & Mayor a 288 m2 & 946 & 11 & 4.210 .200 & 83 \\
\hline Total & Total & 9.094 & $100 \%$ & 5.042 .796 & $100 \%$ \\
\hline
\end{tabular}

Fuente: elaboración propia a partir de la cartografía predial catastral IGAC vigencia 2009.

13 De acuerdo con el estudio adelantado por estas autoras, el tipo edificatorio predominante en las viviendas, tanto para desarrollos planificados como espontáneos, se realiza en parcelas de proporción 1:2, de $6 \mathrm{~m}$ de frente por $12 \mathrm{~m}$ de fondo para un área de lote de $72 \mathrm{~m}^{2}$. 
Figura No. 6. Porcentajes de parcelas y superficie ocupada por rangos de área en la ciudad de Madrid.

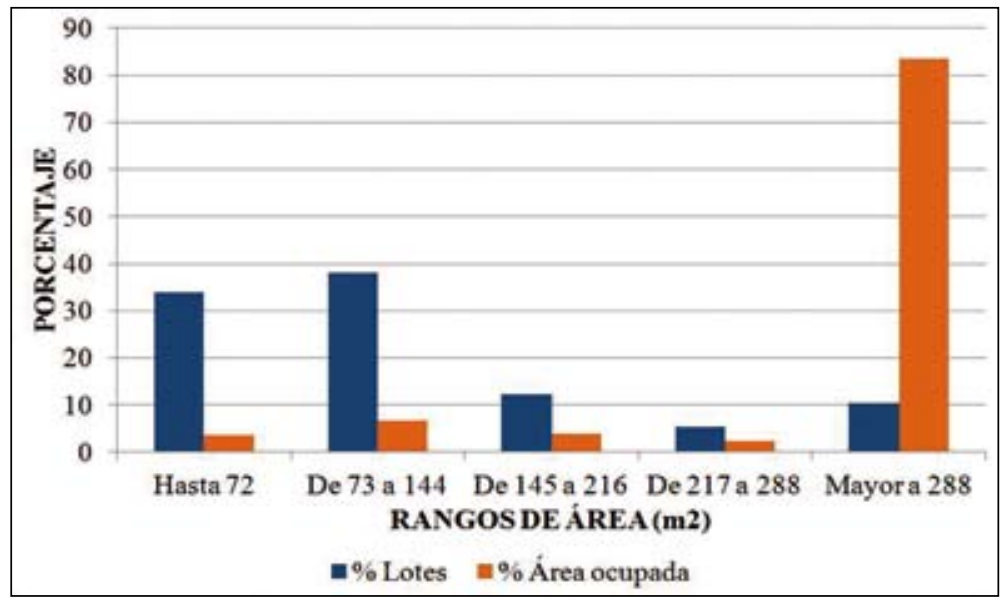

Fuente: elaboración propia a partir de la cartografía predial catastral IGAC vigencia 2009.

La cuantificación de superficies ocupadas de acuerdo con los diferentes usos del suelo en la ciudad permitió identificar tres usos predominantes: los lotes con el $36 \%$ de superficie ocupada, seguido por el uso residencial con el $29 \%$ y el institucional con el $17 \%$. La Figura 8 permite apreciar la distribución espacial de estos usos evidenciando situaciones que determinan la estructura y morfología urbana de la ciudad. La situación más evidente es la presencia de la Base Aérea de la FAC, que figura como el gran polígono de uso institucional en el cuadrante suroccidental. Este polígono constituye una inmensa discontinuidad que interrumpe e impide el avance al sur de la ciudad desarticulando del conjunto urbano los desarrollos que se localizan en su vecindad y que en su mayoría son residenciales. Por otra parte, se observa que la gran mayoría de predios con uso comercial se localizan sobre la Troncal de Occidente. La zona norte de la ciudad se caracteriza por la presencia de grandes superficies sin ocupar, que representan casi una tercera parte del área urbana, mientras que una importante cantidad de predios residenciales se ubica al sur en inmediaciones a la Base Aérea de la FAC. La zona industrial, que ocupa el $8 \%$ de superficie, se encuentra dispersa en grandes extensiones que se localizan al norte de la ciudad. 
Figura 7. Superficie ocupada por diferentes usos del suelo en la ciudad de Madrid.

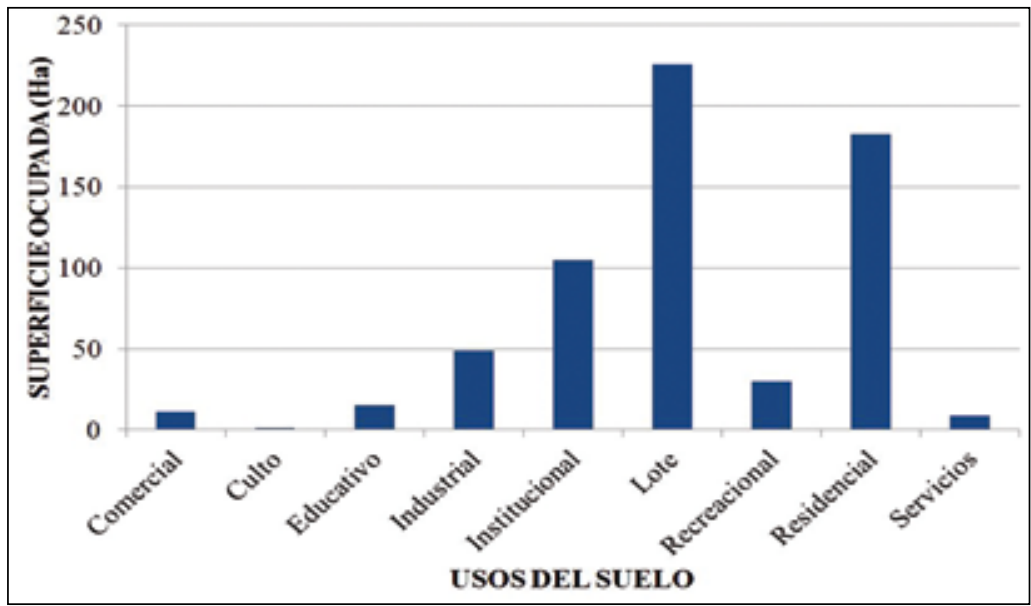

Fuente: elaboración propia a partir de la cartografía del PBOT de Madrid 2004.

Figura 8. Localización de los usos del suelo en la ciudad de Madrid.

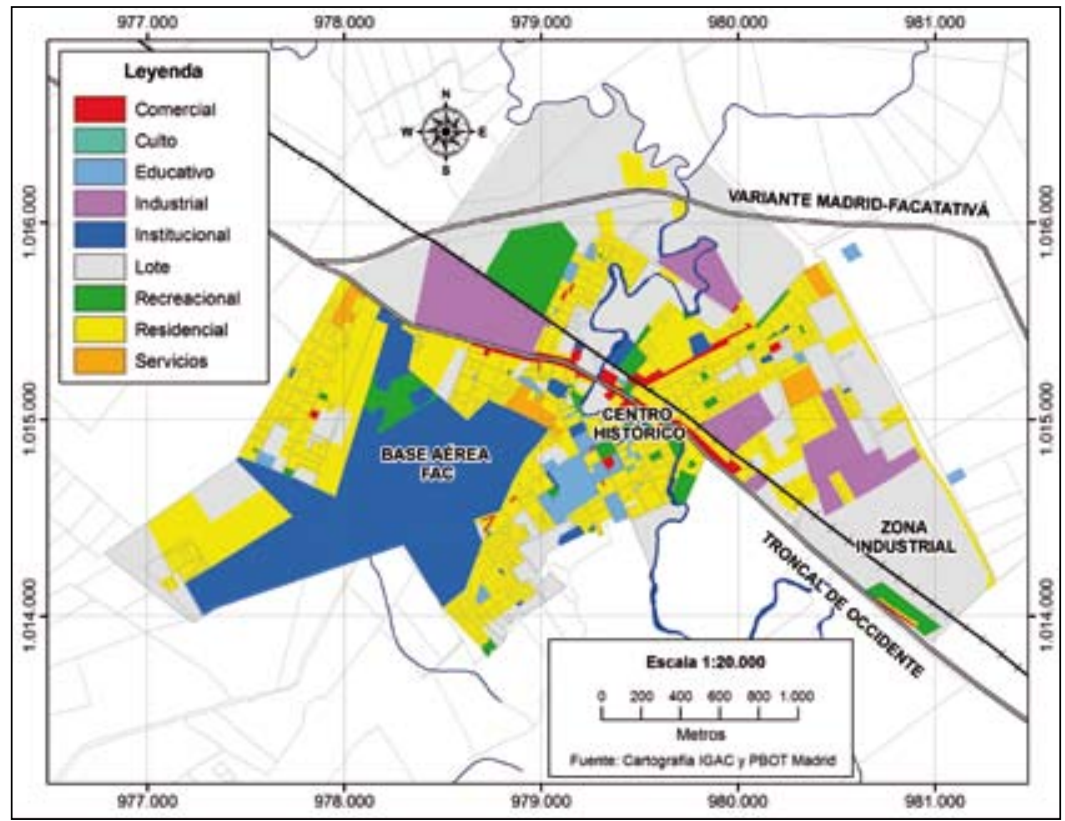

Fuente: elaboración propia a partir de la cartografía del PBOT de Madrid 2004. 


\subsection{Discusión de resultados}

El crecimiento urbano de la ciudad de Madrid en las cuatro últimas décadas se ha caracterizado por la aparición de barrios cuya articulación al conjunto urbano es lograda a través de vías barriales que se conectan a la Troncal de Occidente, eje articulador de la expansión urbana en la ciudad. Estos barrios se emplazan de forma discontinua obedeciendo a un patrón tipo "salto de rana", lo que ocasiona la aparición de espacios vacíos entre los diferentes desarrollos. La tipología morfológica predominante en estos barrios es el plano en forma de damero o cuadrícula la cual se desarrolla a partir del eje vial que permite su conexión con la Troncal de Occidente. La malla vial se extiende hasta los límites establecidos para cada barrio, ocasionando que, con la aparición de nuevos desarrollos que ocupan los vacíos urbanos existentes, se generen nuevas estructuras de damero cuya orientación y forma no coincide con la de los desarrollos ya existentes. A esto se suma la existencia de elementos naturales y culturales, los cuales ya fueron mencionados, que actúan como barreras, condicionando la forma y tamaño de los barrios que han venido surgiendo.

La composición de la estructura parcelaria indica altos niveles de subdivisión predial en las zonas residenciales, a tal punto que en la actualidad existe un alto porcentaje de predios que está por debajo de las dimensiones mínimas en las que se desarrolla la vivienda dirigida a sectores populares. Esto sugiere hacinamiento y bajas condiciones de calidad en un importante porcentaje de los predios residenciales que se ubican en la ciudad.

La localización y magnitud de los usos del suelo indica desequilibrio interno. Gran parte de las zonas residenciales se ubican al sur de la ciudad en inmediaciones de la Base Aérea de la FAC. La Figura 8 muestra que en estas zonas, aunque predomina el uso residencial, existe una alta heterogeneidad de usos que pueden asociarse con formas de ocupación discontinuas en el tiempo y el espacio, cuyos vacíos han venido ocupándose sin ningún tipo de regulación. A esto se suman los riesgos que implica la presencia de una pista aérea militar enclavada en una zona predominantemente residencial. En contraste, la zona norte cuenta con grandes espacios por desarrollar. Se destaca además en esta zona la coexistencia de usos residenciales e industriales.

Este proceso se ha dado en un contexto en el que la metropolización de Bogotá ejerce cada vez más presión sobre las dinámicas de los centros urbanos que se localizan en su área 
de influencia. La ciudad de Madrid no es ajena a esta dinámica. La presencia de Bogotá marca de manera cada vez más clara la tendencia al avance del proceso de urbanización del corredor aún rural que separa a Madrid del área metropolitana. Esta tendencia se manifiesta en la densidad de los flujos de transporte y en fenómenos como la conurbación, la localización de usos del suelo asociados con el sector terciario de la economía sobre predios rurales del corredor vial Bogotá-Madrid (parques industriales, bodegas, centros logísticos, oficinas) y la aparición de proyectos de vivienda dirigida a población de estratos altos como es el caso de la urbanización Sabana Pijao, localizada sobre la variante Madrid-Facatativá, al norte de la ciudad.

Este documento es un ejemplo de la vigencia que mantiene la morfología urbana como campo de investigación para explicar la evolución de las ciudades contemporáneas. Atendiendo lo expuesto por Lévy $(2005)^{14}$, se observa la importancia de aplicar enfoques como el estudio del tejido urbano, de los elementos que lo componen (parcela, manzana, sistema viario, espacios libres y espacios construidos)

14 Lévy (2005) propone reorientar el estudio de la morfología urbana pasando de las perspectivas tradicionales de tipo descriptivo a nuevos enfoques de tipo explicativo e interpretativo, por medio del desarrollo de nuevas técnicas y metodologías que le aporten autonomía a este campo de la geografía urbana. y de las causalidades existentes entre estos elementos y la perspectiva funcional (análisis de ciclos económicos, ciclos constructivos y usos del suelo), para comprender los procesos que modelan la ciudad. La aplicación de este tipo de enfoques en una ciudad intermedia como Madrid, claramente influenciada por el crecimiento de Bogotá, presenta un complemento a la mirada tradicional de la metropolización desde la ciudad dominante, aportando elementos para comprender con mayor detalle los impactos de este fenómeno, especialmente en núcleos urbanos de menor jerarquía.

\section{Conclusiones}

La expansión física y la forma urbana de Madrid son el resultado de la influencia de múltiples factores de tipo local y regional, que en diferentes momentos de la historia de la ciudad han determinado los procesos de ocupación de este espacio urbano. El primero de ellos es la situación geográfica del poblado original, el cual dio lugar a la actual ciudad de Madrid, con respecto al antiguo camino Santafé de Bogotá-Honda, hoy Troncal de Occidente. Gracias a esta condición, Madrid se ha favorecido a lo largo de la historia de las ventajas que derivan al localizarse a corta distancia del principal centro urbano del país. Otro factor determinante que ha influido en las manifestaciones físicas del proceso de urbanización de esta ciudad es su emplazamiento en la región. Esta variable, que hace referencia 
a las condiciones naturales del territorio, ha facilitado la expansión horizontal de la ciudad en todas direcciones, a excepción del sitio en que se localiza el cerro Casablanca, gracias a la topografía plana que domina.

La localización de la Base Aérea de la FAC y de grandes industrias como Colcerámica, impactaron de forma definitiva la configuración de la estructura urbana madrileña. La magnitud de los espacios en que se ubicaron estas instalaciones rompió con la secuencia de expansión urbana continua que mantenía la ciudad antes de la década del sesenta, ocasionando la aparición de un nuevo tipo de expansión de patrón discontinuo que Benguigui et al. (2001) denominan "salto de rana". Este patrón de ocupación se reforzó con la llegada de población obrera que se asentó en la ciudad, gracias a las expectativas de empleo resultantes de actividades económicas nacientes como la industria y la floricultura. Las lógicas de ocupación de estos nuevos pobladores propiciaron fenómenos como la urbani- zación ilegal, la "inquilinización” y la fragmentación predial.

El surgimiento de nuevas actividades de bodegaje, logística y vivienda de estratos altos en la ciudad y su entorno inmediato, ponen de manifiesto las tendencias futuras de urbanización de esta ciudad. Como resultado de la influencia metropolitana, Madrid está adquiriendo nuevas funciones urbanas pasando de ser una ciudad dormitorio a ser un núcleo de alto valor estratégico para el sistema urbano de la Sabana de Bogotá, en el que gradualmente se asientan actividades económicas de gran importancia para la región así como un importante porcentaje de población. Esta situación representa un gran potencial de desarrollo para el municipio y sus pobladores, pero a la vez plantea grandes retos para la administración municipal. Estos retos básicamente se resumen en el hecho de tener que corregir el desorden y desequilibrio que actualmente existen en el tejido urbano madrileño en una ciudad cuya dinámica urbana tiende a crecer y a hacerse aun más compleja. 


\section{Literatura citada}

Alcaldía de Madrid, Cundinamarca. (1969). Plan de desarrollo del municipio de Madrid. Madrid, Colombia.

Alcaldía de Madrid, Cundinamarca. (2000). Plan básico de ordenamiento territorial. Madrid, Colombia.

Allain, R. (2004). Morphologie urbaine: géopraphie, aménagement et architecture de la ville. Paris: Colin.

Beaujeau-Garnier, J. \& Chabot, G. (1970). Tratado de geografía urbana. Barcelona: VicensVives.

Benguigui, L., Czamananski, D. \& Marinov, M. (2001). City growth as a leap-frogging process: an application to the Tel-Aviv metropolis. Urban studies 38 (10), 1819-1839. Recuperado el 1 de julio de 2011, de http://usj.sagepub.com/content/38/10/1819. full.pdf + html.

Capel, H. (1975). La definición de lo urbano. En Estudios Geográficos, 138-139 (número especial de "Homenaje al Profesor Manuel de Terán"), 265-301.

Capel, H. (2002). La morfología de las ciudades. Sociedad, cultura y paisaje urbano, I. Barcelona: Del Serbal.

Carter, H. (1974). El estudio de la geografía urbana. Madrid: Instituto de Estudios de Administración Local.

Delgado, C. (2004). Camino nacional Honda-Santafé de Bogotá: transporte, economía y desarrollo. En Apuntes del Cenes, XXIV fasc.N/A, 199-227. Tunja: Universidad Pedagógica y Tecnológica de Colombia.

Extractos de los diarios de Humboldt. Recuperado en febrero de 2011, de http://www.banrepcultural.org/blaavirtual/exhibiciones/humboldt.

Gauthier, P. \& Jason, G. (2006). Mapping urban morphology: a classification scheme for interpreting contributions to the study of urban form. Recuperado el 3 de Febrero de 2011, de http://www.arch.mcgill.ca/prof/luka/urbandesignhousing/602fall2010/ readings.

González, R. (2007). Implicaciones de la floricultura en las transformaciones espaciales de Madrid (Cundinamarca) a partir de 1970. Tesis presentada para optar al título de Magíster en Geografía, no publicada. UPTC, IGAC, Bogotá.

Instituto Geográfico Agustín Codazzi IGAC. (1974). Estudio integrado de la Sabana de Bogotá, Municipio de Madrid. Bogotá: Subdirección de investigación y divulgación geográfica.

Instituto Geográfico Agustín Codazzi IGAC. (2005). Carta General Hoja No. 227, E100K, Noroccidente de Bogotá. Bogotá: IGAC. 
Lévy, A. (2005). Formes urbaines et significations: Revisiter la morphologie urbaine. Espaces et Sociétes, 3 (122), 25-48. Recuperado el 8 de Julio de 2011, de http://www.cairn. info/article.php?ID_ARTICLE $=$ ESP_122_0025.

Mesa de Planificación Regional Bogotá-Cundinamarca, Departamento de Asuntos Económicos y Sociales de las Naciones Unidas UNDESA/UNCRD. (2005). De las ciudades a las regiones, Desarrollo regional integrado en Bogotá-Cundinamarca. Volumen 2: Estudios y procesos. Bogotá: UNDESA.

Molina, H. (2003). Dinámica demográfica y estructura funcional de la región BogotáCundinamarca 1973-2020. En UNDESA/UNCRD. (2005). De las ciudades a las regiones, desarrollo regional integrado en Bogotá-Cundinamarca. Volumen 1: Soporte técnico. Bogotá: UNDESA.

Montañez, G. (1992). ¿Hacia dónde va la Sabana de Bogotá? Modernización, conflicto, ambiente y sociedad. Bogotá: SENA-UNAL.

Pérez, A. (2000). La estructura ecológica principal de la Sabana de Bogotá. Bogotá: Sociedad Geográfica Colombiana.

Puyol, R., Estébanez, J. \& Méndez, R. (1988). Geografia humana. Madrid: Cátedra.

Suárez, E. A. (1990). Análisis socio administrativo del municipio de Madrid. Tesis presentada para optar al título de Administrador Público, no publicada. EAN. Bogotá.

Tarchópulos, D. \& Ceballos, O. (2005). Patrones urbanísticos y arquitectónicos en la vivienda dirigida a sectores de bajos ingresos en Bogotá. Bogotá: Pontificia Universidad Javeriana.

Torres, C. A. (2005). Dualidad formal informal, el modelo de producción de la ciudad colombiana. Una mirada desde los años 90. En Rincón, A. (Ed). Espacios urbanos no con-sentidos. Legalidad e ilegalidad en la producción de la ciudad, (pp. 123152). Medellín: UNAL.

UNESCO. (1999). Ciudades intermedias y urbanización mundial. Lleida, España. Recuperado el 12 de abril de 2011, de http://www.unesco.org/most/ciudades.pdf.

Van Der Hammen, T. (1998). Plan ambiental de la cuenca alta del río Bogotá. Bogotá: Corporación Autónoma Regional CAR.

Velandia, R. (1971). Historia geopolítica de Cundinamarca. Bogotá: Extensión Cultural de la Gobernación de Cundinamarca.

Zárate, A. (1991). El espacio interior de la ciudad. Madrid: Síntesis.

Zárate, A. (1992). El mosaico urbano. Organización interna y vida en las ciudades. Madrid: Cincel Kapelusz.

Recepción: 04 de agosto de 2011

Evaluación: 16 de agosto de 2011

Aprobación: 09 de septiembre de 2011 
